\title{
Simulation of Shrinkage Porosity Formation During Alloy Solidification
}

\begin{abstract}
VAHID KHALAJZADEH and CHRISTOPH BECKERMANN
Porosity due to solidification shrinkage is a troublesome defect in metal casting. It results in low yields and increased costs in production and limits the performance of cast components in service. By reliably predicting porosity in casting process simulation, porosity can be minimized or eliminated. Here, a new model for simulating the formation of shrinkage porosity during solidification is presented. The model is based on the recent discovery that shrinkage porosity nucleates and grows in regions of a casting where the solid fraction is the lowest. It calculates the feeding flows and pressure distribution in the liquid while accounting for the density variation during cooling and solidification. It predicts the location, extent and amount of all types of shrinkage porosity in a casting, including riser pipes and large internal holes, surface sinks, and distributed micro-shrinkage. Porosity predictions are presented for simple casting geometries and for a more complex Mn-steel experimental casting. The comparisons to the observations made in the experimental casting demonstrate the capability of the model to accurately predict the various types of shrinkage porosity. Numerical studies are performed to investigate the sensitivity of the predictions to various model parameters.
\end{abstract}

https://doi.org/10.1007/s11661-020-05699-z

(C) The Minerals, Metals \& Materials Society and ASM International 2020

\section{INTRODUCTION}

SOLIDIFICATION shrinkage related porosity is a defect of foremost concern in the metal casting industry. Shrinkage porosity reduces the mechanical performance of cast parts, causes leaks, and is generally unacceptable to customers of foundries. ${ }^{[1-3]}$ It occurs when the liquid melt can no longer feed the volume contraction of a metal alloy during solidification. Usually, shrinkage porosity is combatted by adding so-called risers or feeders to the mold during casting. Nevertheless, when the feeding path from the risers is cut off, surface sinks and internal holes will develop. Figure 1 shows examples of such shrinkage related defects. For more than 30 years now, computer casting simulation software has been used to design casting rigging systems that avoid porosity while keeping the number and size of risers and other feeding aids to a minimum. However, the mathematical models underlying such simulations are often highly simplified and do not accurately predict the location, extent, type, and amount of shrinkage porosity in a casting.

VAHID KHALAJZADEH and CHRISTOPH BECKERMANN are with the Department of Mechanical Engineering, University of Iowa, Iowa City, IA 52242. Contact e-mail: becker@engineering.uiowa.edu Manuscript submitted December 4, 2019.

Article published online March 10, 2020
The early studies by Piwonka and Flemings ${ }^{[4]}$ and Kubo and Pehlke ${ }^{[5]}$ have inspired numerous advancements in understanding and modeling of porosity formation during alloy solidification. The advances include but are not limited to: applying three-dimensional (3D) models for the calculation of the liquid pressure, feeding velocity, and porosity distributions during solidification, including the effects of dissolved gases, using dynamic mesh refinement algorithms for the semi-solid mushy zone, tracking the liquid surface movement due to solidification shrinkage with a volume-of-fluid (VOF) method, and developing physics-based theories for the onset of pore nucleation. ${ }^{[6-15]}$ Summaries of these previous studies can be found in review papers. ${ }^{[16-18]}$ Although the modeling of shrinkage porosity in castings has progressed significantly, a model that simultaneously predicts sinks and pipes emanating from a surface, internal macro-porosity holes, and distributed microporosity is still lacking. Aside from the fact that previous models are not sufficiently comprehensive for use in casting simulation, another main shortcoming is the lack of a realistic treatment of when and where shrinkage porosity nucleates and how it spreads.

The present paper is a continuation of the recent study by Khalajzadeh et al., ${ }^{[19]}$ who investigated the formation of shrinkage porosity in an aluminum alloy wedge casting using real-time video radiography. The images in the recorded video were processed to obtain quantitatively accurate pore volume fraction fields in the 


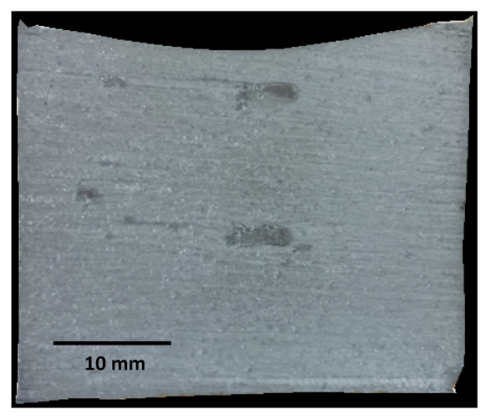

(a)

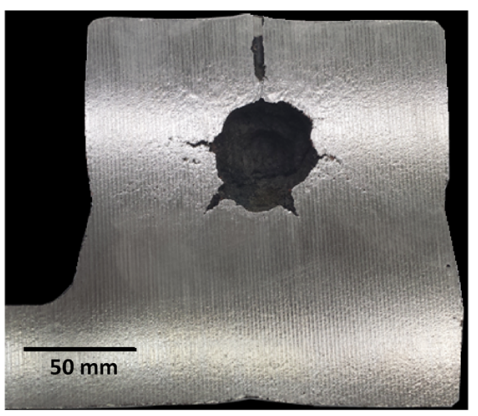

(b)



(c)

Fig. 1-Surface sinks and internal porosity defects caused by solidification shrinkage: $(a)$ a depression or sink on the top surface of a cast aluminum block, $(b)$ internal macro-shrinkage hole in a steel casting, and $(c)$ distributed internal micro-shrinkage porosity.

casting as a function of time, spanning from nucleation and growth to final distribution. Their results indicate that the shrinkage porosity in the wedge evolves in two stages. Sinks start forming first at those locations on the surface where the solid fraction is lowest, with the hydrostatic head pressure resisting their development to some degree. The sinks continue to grow until the entire surface of the wedge becomes coherent and rigid, which was determined to occur at a solid volume fraction of 40 pct. Then, internal porosity nucleates in the thermal center of the wedge where the solid fraction is the lowest, with the hydrostatic head pressure again playing a minor role. It continues to grow and spread until the wedge is fully solidified. The total solidification shrinkage of the aluminum alloy was divided about equally between surface sinks (Figure 1(a)) and internal porosity (Figure 1(c)). Based on the experimental observations, a pore nucleation and growth model was developed to predict the evolution of the shrinkage porosity. Excellent agreement was obtained between measured and predicted surface and internal porosity patterns during solidification. This model provides the first realistic treatment of the nucleation, growth and spreading of shrinkage porosity by accounting for the important effects of solid fraction and hydrostatic pressure. However, it was noted that for the model of Khalajzadeh et al. ${ }^{[19]}$ to be useful for general casting geometries with multiple porosity containing regions it must be extended to include calculation of the dynamic pressures and feeding flows. This is the purpose of the present part of the study. Transient temperature and solid fraction fields are obtained from standard casting simulation software. A modified form of the porosity nucleation and growth model of Khalajzadeh et al. ${ }^{[19]}$ is then supplemented by equations describing the shrinkage driven feeding flow and pressure distribution, leading ultimately to predictions of the pore volume fraction evolution throughout a casting. First, certain features of the model are demonstrated using simple casting geometries. The model is then applied to a relatively complex shaped experimental Mn-steel casting. The casting contained a large shrinkage pipe in the riser, an open shrinkage hole in the center of an end block, and distributed centerline shrinkage in a plate section. The experimental results are compared to the model predictions and a numerical study is performed to investigate the sensitivity of the results to the model parameters. Porosity due to dissolved gases, although important for some alloys, is not considered.

\section{COMPUTATIONAL MODEL}

A schematic diagram of a solidifying casting volume is shown in Figure 2. Multiple regions of porosity can form simultaneously. The boundary conditions at the casting surface and on the porosity containing regions are indicated in Figures 2(a) and (c), respectively. The model allows for a representative elementary volume to be composed of three phases: solid (s), liquid $(\ell)$ and porosity (p). Using " $g$ " to denote the volume fraction of a given phase, and the phases indicated by subscripts, the volume fractions must satisfy $g_{\ell}+g_{\mathrm{s}}+g_{\mathrm{p}}=1$, as shown schematically in Figure 2(b). Key assumptions in the model are:

- Solid and porosity phases are stationary, i.e., they cannot move during solidification; however, solid may be replaced by porosity if the solid fraction is below a certain coherency limit (see Eq. [19]).

- Flow of the liquid melt is driven by density changes only; buoyancy-driven flow during solidification is neglected.

- Density is temperature-dependent above the solidus temperature and assumed constant when the metal is solid; the density of the gases inside of shrinkage pores is neglected.

The governing equations for the feeding flow and the procedures used for predicting the evolution of the pore volume fraction are given in the following sub-sections. The model assumes that the results from a standard thermal simulation of the casting are available. This includes the evolution of the temperature and solid fraction fields throughout the solidification process. The heat transfer is assumed to be unaffected by the porosity.

\section{A. Continuity and Momentum Equations}

Mass is conserved according to a mixture continuity equation given by 


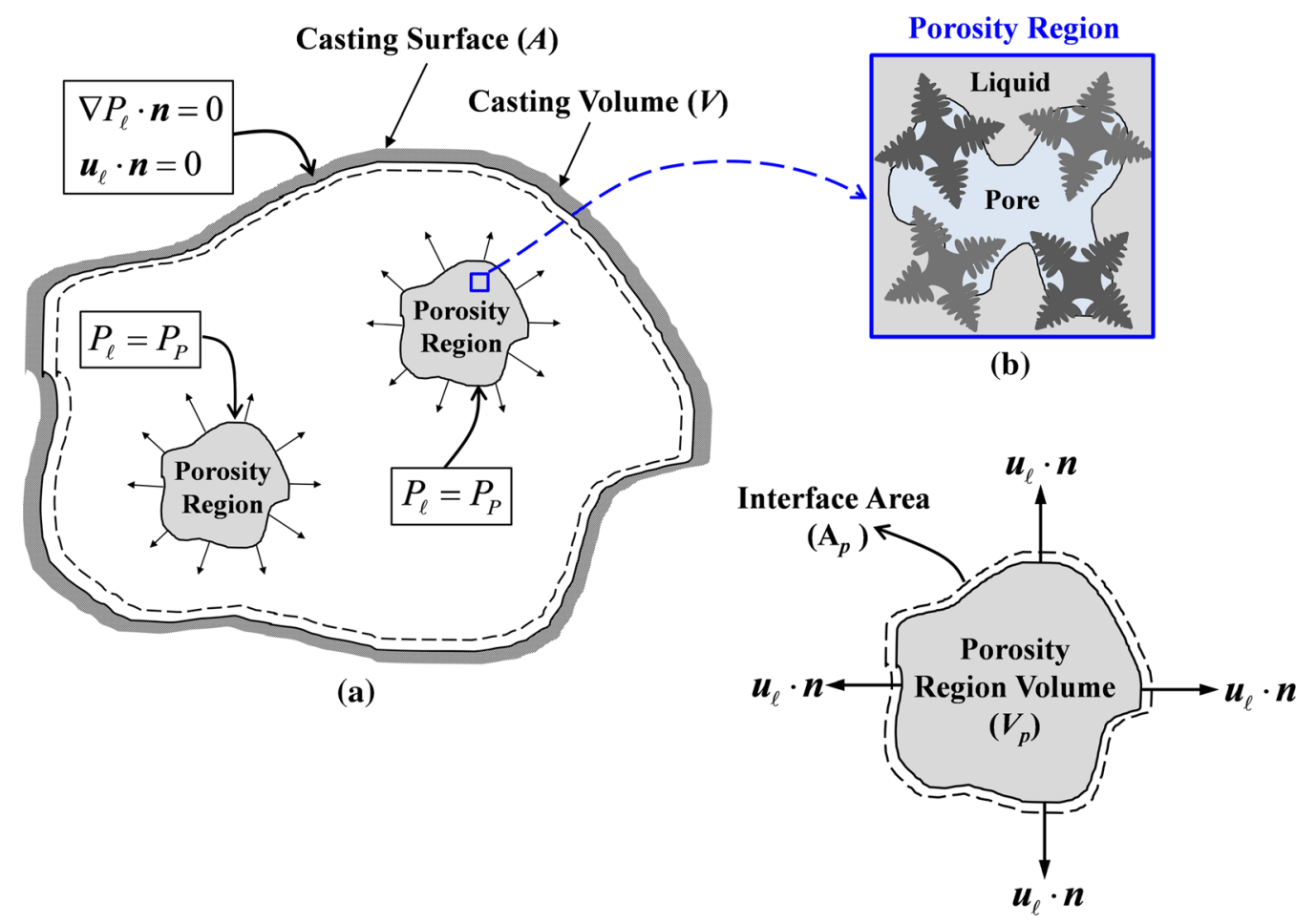

(c)

Fig. 2-Schematic diagram of a solidifying casting with porosity: (a) casting volume with multiple porosity containing regions, (b) representative elementary volume containing solid, liquid and porosity, and $(c)$ single porosity containing region (Color figure online).

$$
\frac{\partial \bar{\rho}}{\partial t}+\nabla \cdot\left(\rho_{\ell} \boldsymbol{u}_{\ell}\right)=0
$$

where $\bar{\rho}$ is the mixture density (see Section II-C for more detail), $t$ is time, $\rho_{\ell}$ is the liquid density, and $\boldsymbol{u}_{\ell}$ is the superficial liquid velocity vector. The feeding flow in the mushy zone is assumed to be a creeping flow in a porous medium of variable permeability. For such flows the momentum equation is given by Darcy's law,

$$
\nabla P_{\ell, \mathrm{t}}=-\frac{\mu_{\ell} \boldsymbol{u}_{\ell}}{K}+\rho_{\ell} \boldsymbol{g}
$$

where $P_{\ell, \mathrm{t}}$ is the total liquid pressure, $\mu_{\ell}$ is the dynamic viscosity of the melt, $K$ is the permeability of the mush, and $\boldsymbol{g}$ is the gravity vector. Equation [2] is also used as the momentum equation for the flow in the pure liquid region of the casting, where the solid fraction is zero, by setting the permeability to a suitably large value, $K_{\max }$. A value of $1 \times 10^{-6} \mathrm{~m}^{2}$ was found to cause a negligibly small pressure drop in the single phase liquid regions and was used throughout this study. The total liquid pressure $\left(P_{\ell, \mathrm{t}}\right)$ is the sum of the dynamic liquid pressure $\left(P_{\ell}\right)$ and the hydrostatic pressure $\left(P_{\mathrm{h}}\right)$, i.e., $P_{\ell, \mathrm{t}}=P_{\ell}+P_{\mathrm{h}}$. Applying the gradient operator to this equation gives

$$
\nabla P_{\ell, \mathrm{t}}=\nabla P_{\ell}+\nabla P_{\mathrm{h}}
$$

where the hydrostatic pressure gradient is given by $\nabla P_{\mathrm{h}}=\rho_{\ell}$ g. Combining Eqs. [2] and [3], the gravity term cancels and Eq. [2] simplifies to

$$
\nabla P_{\ell}=-\frac{\mu_{\ell} \boldsymbol{u}_{\ell}}{K}
$$

The permeability of the mush is a function of solid fraction $\left(g_{\mathrm{s}}\right)$ according to the Kozeny-Carman equation

$$
K=K_{0} \frac{\left(1-g_{\mathrm{s}}\right)^{3}}{g_{\mathrm{s}}^{2}}
$$

where $K_{0}$ is an adjustable constant permeability coefficient. To avoid division by zero in Eq. [4], a suitable minimum allowable value for the permeability, $K_{\min }$, is introduced. The effect of variations in $K_{0}$ and $K_{\min }$ is explored below in a parametric study.

A constant reference liquid pressure is needed to determine a solution of Eq. [4]. Here it is assumed that $P_{\ell}$ within regions having porosity is equal to the pore pressure, i.e., $\left.P_{\ell}\right|_{\text {Porosity }}=P_{\mathrm{p}}$, implying that surface tension effects are neglected. The value of the pore pressure $P_{\mathrm{p}}$ depends on the location in the casting. At an atmosphere-metal interface the pore pressure is assumed to be atmospheric, i.e., $P_{\mathrm{p}}=P_{\text {atm }}$; at a mold-metal interface it is equal to some pre-defined mold atmosphere pressure, i.e., $P_{\mathrm{p}}=P_{\text {mold }}$; and for internal porosity, the pores are assumed to be vacuum (negligible density), i.e., $P_{\mathrm{p}}=0$. By forcing the dynamic pressure to a uniform value, the liquid velocities vanish inside of porosity forming regions.

Assuming for simplicity that $\rho_{\ell}$ in Eqs. [1] and [2] is constant, Eqs. [1] and [4] can be combined to form an 
equation for determining the pressure $P_{\ell}$ throughout the casting during solidification

$$
\nabla \cdot\left(\frac{K}{\mu_{\ell}} \nabla P_{\ell}\right)=\frac{1}{\rho_{\ell}} \frac{\partial \bar{\rho}}{\partial t}
$$

As shown in Figure 2, the boundary condition for the above equation is given by a zero-mass flux condition at the casting surface, which can be written as $\left(\boldsymbol{u}_{\ell}\right.$. $\boldsymbol{n})_{\text {Surface }}=\left(\nabla P_{\ell} \cdot \boldsymbol{n}\right)_{\text {Surface }}=0$, where $\boldsymbol{n}$ is the normal vector on the surface. Using this boundary condition and forcing the pressure in the porosity containing regions to $\left.P_{\ell}\right|_{\text {Porosity }}=P_{\mathrm{p}}$, the pressure distribution $P_{\ell}$ in the casting can be determined by solving Eq. [6]. The expanded form of Eq. [6] in 3D Cartesian coordinates is given by

$$
\frac{\partial}{\partial x}\left(\frac{K}{\mu_{\ell}} \frac{\partial P_{\ell}}{\partial x}\right)+\frac{\partial}{\partial y}\left(\frac{K}{\mu_{\ell}} \frac{\partial P_{\ell}}{\partial y}\right)+\frac{\partial}{\partial z}\left(\frac{K}{\mu_{\ell}} \frac{\partial P_{\ell}}{\partial z}\right)=\frac{1}{\rho_{\ell}} \frac{\partial \bar{\rho}}{\partial t}
$$

which is discretized and solved using a finite volume approach. After $P_{\ell}$ is determined, the total pressure $\left(P_{\ell, \mathrm{t}}\right)$ is calculated according to

$$
P_{\ell, \mathrm{t}}=P_{\ell}+P_{\mathrm{h}}
$$

where the hydrostatic pressure is given by

$$
P_{\mathrm{h}}=\rho_{\ell} g\left(z_{\max }-z\right)
$$

in which $\left(z_{\max }-z\right)$ is the head height relative to a maximum elevation of $z_{\max }$. The velocity distribution in the casting due to solidification shrinkage is then determined by solving Eq. [4]. The velocity components in Cartesian coordinates are given by

$$
u_{\ell}=-\frac{K}{\mu_{\ell}}\left(\frac{\partial P_{\ell}}{\partial x}\right) \quad v_{\ell}=-\frac{K}{\mu_{\ell}}\left(\frac{\partial P_{\ell}}{\partial y}\right) \quad w_{\ell}=-\frac{K}{\mu_{\ell}}\left(\frac{\partial P_{\ell}}{\partial z}\right)
$$

where $u_{\ell}, v_{\ell}, w_{\ell}$ are the superficial liquid velocity components in the $x, y, z$ directions.

\section{B. Pore Nucleation Model}

The present pore nucleation model is the same as the one developed by Khalajzadeh et al., ${ }^{[19]}$ except that the hydrostatic pressure $\left(P_{\mathrm{h}}\right)$ is replaced by the total liquid pressure $\left(P_{\ell, \mathrm{t}}\right)$. Nonetheless, a brief summary of the model is provided here for completeness. Khalajzadeh et al. ${ }^{[19]}$ found that surface sinks and internal porosity do not evolve at the same time. Surface sinks develop first and internal porosity forms in a second stage. The transition between the two stages is modeled by introducing the critical solid fraction $g_{\mathrm{s} \text { sur }}$. When the solid fraction at the surface is below this critical value, a surface sink can form. Once the solid fraction everywhere on the surface is above $g_{\mathrm{s}, \mathrm{sur}}$, the surface becomes rigid and internal porosity can nucleate.
The initiation of surface sinks and internal porosity is modeled using the same method. The pore nucleation model is based on the inequality form of the Young-Laplace equation, which is given in the present study by

$$
P_{\mathrm{p}}-P_{\ell, \mathrm{t}} \geq P_{\sigma}
$$

where $P_{\sigma}$ is a capillary pressure due to surface tension. As found by Khalajzadeh et al., ${ }^{[19]}$ the capillary pressure $P_{\sigma}$ in Eq. [11] can be taken as a linear function of the solid fraction as

$$
P_{\sigma}=P_{\sigma, 0} \times g_{\mathrm{s}}
$$

where $P_{\sigma, 0}$ is a reference capillary pressure at a solid fraction of unity. The reference pressure $P_{\sigma, 0}$ is an adjustable parameter that controls the absolute magnitude of the capillary term and the relative importance of the total liquid and capillary pressures. Equation [12] implies that pore nucleation is favored at locations where the solid fraction is the lowest, since there the resisting effect of the capillary pressure is the smallest. As in Khalajzadeh et al., ${ }^{[19]}$ the Young-Laplace inequality is implemented in the model through a so-called $\Pi$ parameter, which is defined as

$$
\Pi=P_{\ell, \mathrm{t}}-\left(P_{\mathrm{p}}-P_{\sigma}\right)
$$

Porosity is thus nucleated where the $\Pi$ parameter is the lowest. To make the nucleation calculations numerically robust, porosity is nucleated where the $\Pi$ value falls within a small dimensionless interval $\varepsilon_{\text {nuc }}$ according to:

$$
\left|\frac{\Pi-\Pi_{\min }}{\Pi_{\max }-\Pi_{\min }}\right|<\varepsilon_{\mathrm{nuc}}
$$

where $\Pi_{\min }$ is the instantaneous minimum $\Pi$ value in the computational domain and $\Pi_{\max }$ is the maximum possible $\Pi$ value. Using $\Pi_{\min }$ instead of zero ensures that there is always at least one computational cell that has porosity. Physically, the adjustable parameter $\varepsilon_{\text {nuc }}$ controls over how large a region the porosity nucleates.

\section{Pore Growth Model}

As shown in Figure 2(a), multiple regions of porosity can nucleate and grow in a casting simultaneously. Each region is a grouping of connected computational cells having $g_{\mathrm{p}}>0$. These cells are identified using the search algorithm of Reference 20. The volume and surface area of each porosity forming region are denoted by $V_{\mathrm{p}}$ and $A_{\mathrm{p}}$, respectively, as shown in Figure 2(c). The average pore growth rate $\left(d \bar{g}_{\mathrm{p}} / d t\right)$ for each porosity containing region is calculated by integrating the mass conservation equation, Eq. [1], over $V_{\mathrm{p}}$. Using the divergence theorem for the second term in Eq. [1] yields

$$
\int_{V_{\mathrm{P}}}\left(\frac{\partial \bar{\rho}}{\partial t}\right) d V+\int_{A_{\mathrm{P}}}\left(\rho_{\ell} \boldsymbol{u}_{\ell}\right) \cdot \boldsymbol{n} d A=0
$$


The three-phase mixture density, $\bar{\rho}$, is defined as $\bar{\rho}=$ $\rho_{\mathrm{s}} g_{\mathrm{s}}+\rho_{\ell} g_{\ell}+\rho_{\mathrm{p}} g_{\mathrm{p}}$. This density should be distinguished from the solid-liquid mixture density in the absence of porosity, which is given by $\bar{\rho}^{S L}=\rho_{\mathrm{s}} g_{\mathrm{s}}^{S L}+\rho_{\ell} g_{\ell}^{S L}$, where $g_{\mathrm{s}}^{S L}+g_{\ell}^{S L}=1$. The density $\bar{\rho}^{S L}$ is assumed to be known as a function of temperature for a given metal alloy. Assuming that $g_{\mathrm{s}}^{S L}=g_{\mathrm{s}}$ and $\rho_{\mathrm{p}} \ll \rho_{\mathrm{s}}, \rho_{\ell}$ yields the following equation for the three-phase density

$$
\bar{\rho}=\bar{\rho}^{S L}-\rho_{\ell} g_{\mathrm{p}}
$$

Assuming that $\rho_{\ell}$ in Eq. [16] is constant and that the pore growth rate is uniform within each $V_{\mathrm{p}}$, substitution of Eqs. [16] into [15] results in

$$
\frac{d \bar{g}_{\mathrm{p}}}{d t}=\frac{1}{\rho_{\ell} V_{\mathrm{p}}} \int_{V_{\mathrm{P}}}\left(\frac{\partial \bar{\rho}^{S L}}{\partial T}\right) d V+\frac{1}{V_{\mathrm{P}}} \int_{A_{\mathrm{P}}} \boldsymbol{u}_{\ell} \cdot \boldsymbol{n}_{\ell} d A
$$

Equation [17] allows for the calculation of the average pore growth rate in each porosity forming region from the knowledge of the average solid-liquid mixture density variation within $V_{\mathrm{p}}$ and the feeding flow velocities normal to the boundary $A_{\mathrm{p}}$. In Khalajzadeh et al. ${ }^{[19]}$ the integration in Eq. [17] was carried out over the entire casting volume and the second term on the right-hand side of Eq. [17] was not included, because all porosity was assumed to grow in a single region and the feeding velocities were not calculated. Here, Eq. [17] is applied separately to each porosity forming region. Note that it is permissible to use the mass conservation equation to obtain the pore volume fraction, because the liquid pressure in the porosity forming regions is forced to a known pore pressure (see Section II-A). The average porosity increase from Eq. [17] is then evenly distributed over all active computational cells within each $V_{\mathrm{p}}$ to determine the local time rate of change in the pore fraction according to

$$
\frac{d g_{\mathrm{p}}}{d t}=\left(d \bar{g}_{\mathrm{p}} / d t\right) / g_{\text {active }}
$$

where $g_{\text {active }}=V_{\text {active }} / V_{\mathrm{P}}$ is the volume fraction of active cells in a porosity forming region, in which $V_{\text {active }}$ is the total volume of all active cells within $V_{\mathrm{p}}$. An active cell is defined as a cell where porosity has nucleated and is currently growing.

The pore volume fraction is typically allowed to increase until a computational cell becomes empty of liquid, i.e., when $g_{\mathrm{s}}+g_{\mathrm{p}}=1$. This condition, however, would result in the local pore fraction never being able to approach unity. A pore fraction of unity occurs, for example, when a surface sink or internal hole is free of solid. For the pore volume fraction to reach unity, the previously grown solid at that location must have moved or been pushed away by the growing porosity, which is frequently referred to as mass feeding. As in Khalajzadeh et al. ${ }^{119]}$ mass feeding is modeled indirectly by introducing an adjustable parameter called the coherency solid fraction $g_{\mathrm{s}, \mathrm{coh}}$. The maximum pore volume fraction, $g_{\mathrm{p}, \max }$, is then given by

$$
g_{\mathrm{p}, \max }= \begin{cases}1 & \text { for } \quad g_{\mathrm{s}}<g_{\mathrm{s}, \mathrm{coh}} \\ 1-g_{\mathrm{s}} & \text { for } \quad g_{\mathrm{s}} \geq g_{\mathrm{s}, \mathrm{coh}}\end{cases}
$$

The two critical solid fractions $g_{\mathrm{s}, \mathrm{sur}}$ and $g_{\mathrm{s}, \mathrm{coh}}$ take typically the same value. ${ }^{[19]}$ When a computation cell reaches the maximum pore fraction, it is designated as inactive.

Once a pore forming region has no more active cells, but solidification is not yet complete, porosity can spread beyond the originally nucleated volume. As in Khalajzadeh et al.. ${ }^{[19]}$ such spreading of pore forming regions is modeled using the same $\Pi$ parameter introduced in the previous sub-section. Computational cells that neighbor a cell with porosity already present are activated when their $\Pi$ parameter falls within the interval

$$
\left|\frac{\Pi-\Pi_{\min }}{\Pi_{\max }-\Pi_{\min }}\right|<\varepsilon_{\text {layer }}
$$

The small dimensionless number $\varepsilon_{\text {layer }}$ is an adjustable model parameter that controls the rate of spreading of porosity regions. It is emphasized here that the use of the $\Pi$ parameter in both the pore nucleation and growth models was developed in the first part of this study through careful comparisons with real-time video radiography experiments. ${ }^{[19]}$

\section{EXPERIMENTS AND THERMAL SIMULATION}

Casting experiments were performed to provide data for tuning and validating the present porosity simulation model. The experiments involved a high manganese (Mn) steel in a chemically bonded olivine sand mold. The composition of the steel used in the experiments is provided in Table I. The geometry of the casting is shown in Figure 3 and consists of a gating system, a riser, two steps of different thickness, a thinner plate section, and a thick end block. The mold was made of

Table I. Mn-Steel Composition Given in Weight Percent (Wt Pet)

\begin{tabular}{lc}
\hline Element & Amount (Wt Pct) \\
\hline $\mathrm{C}$ & 1.1 \\
$\mathrm{Mn}$ & 13.2 \\
$\mathrm{Si}$ & 0.65 \\
$\mathrm{P}$ & 0.042 \\
$\mathrm{~S}$ & 0.005 \\
$\mathrm{Cr}$ & 0.33 \\
$\mathrm{Ni}$ & 0.11 \\
$\mathrm{Mo}$ & 0.84 \\
$\mathrm{Al}$ & 0.030 \\
$\mathrm{Cu}$ & 0.11 \\
$\mathrm{Ti}$ & 0.00 \\
$\mathrm{Fe}$ & balance \\
\hline
\end{tabular}




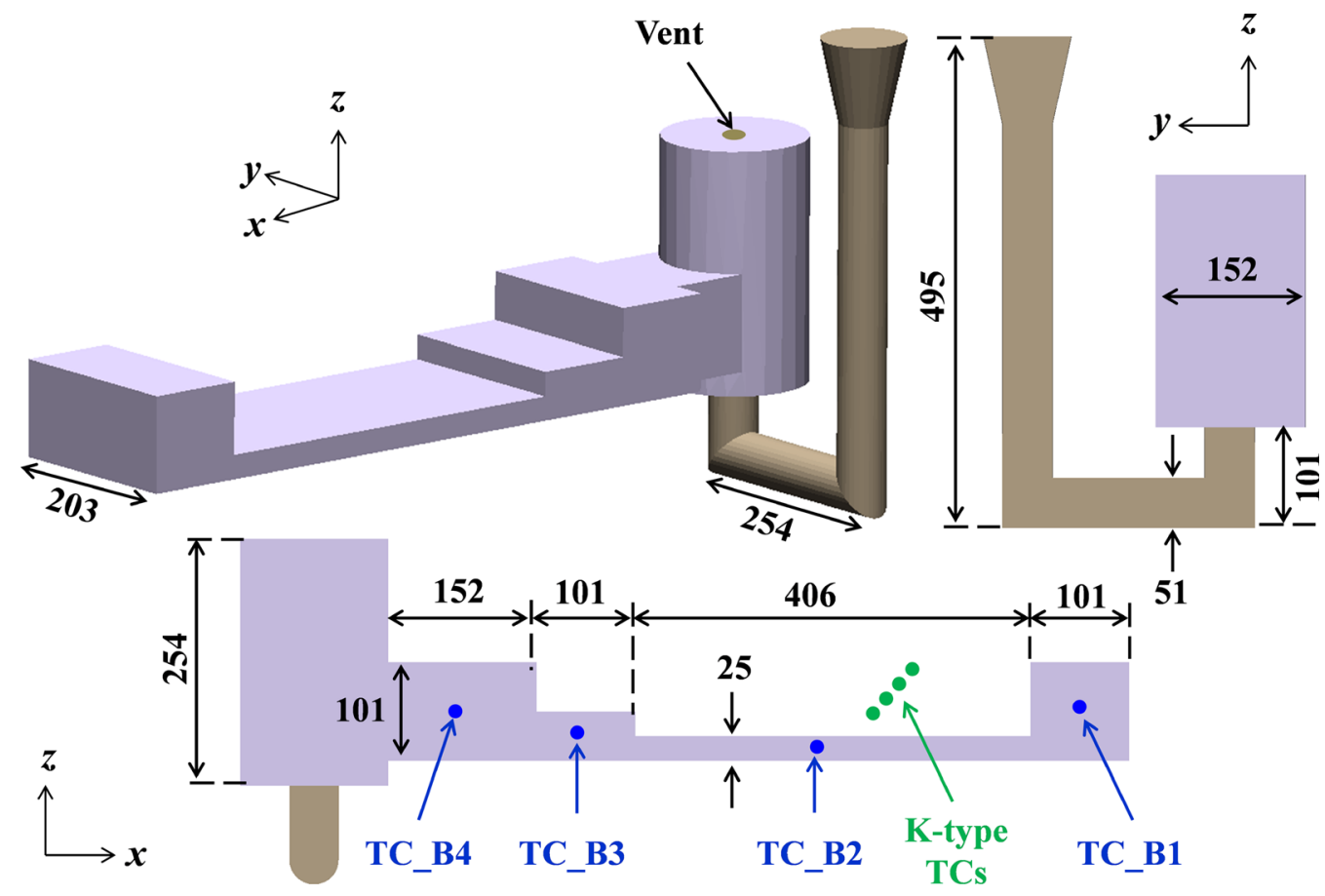

Fig. 3-Mn-steel casting geometry used in the experiments, with TC locations in blue and green; the dimensions are millimeter (mm) (Color figure online).

olivine sand. Use of this sand prevents defects that occur with silica sand, where $\mathrm{SiO}_{2}$ reacts with $\mathrm{MnO}$ in the steel to form a $\mathrm{MnSiO}_{3}$ slag and burn on defects. Thermocouples (TCs) were placed at several locations within the casting and the sand mold. These were used to calibrate the thermal simulations. The type- $K$ thermocouples in the mold were inserted through the top of the cope section at distances of $6,12,18$ and $24 \mathrm{~mm}$ from the mold-metal interface and centered above the plate section. Sheathed type-B thermocouple probes were employed to measure the temperatures of the steel throughout the casting process. These were also inserted through the cope and extended into the center of the steps, the plate section and the end block (see Figure 3). Two identical castings were poured. The temperature measurements made in the two castings agreed well with each other, as shown in Figure 4 (red and blue curves), indicating that the experiments were repeatable.

Thermal simulations of the casting process were performed using a standard software package. ${ }^{[21]}$ The temperature data from the experiments were used to adjust the temperature-dependent thermo-physical properties and other parameters needed in the thermal simulations. First, IDS ${ }^{[23]}$ and JMatPro ${ }^{\mathrm{TM}}{ }^{[24]}$ were used to generate an initial dataset for the temperaturedependent properties, including the solid fraction vs temperature curve. Then, these properties and other simulation settings were varied in a trial-and-error procedure $^{[22]}$ until good agreement between the measured and predicted temperatures was achieved. Analyzing the thermocouple measurements, the solidus and liquidus temperatures of the $\mathrm{Mn}$-steel alloy were found to be $T_{\text {sol }}=1250^{\circ} \mathrm{C}\left(2300^{\circ} \mathrm{F}\right)$ and $T_{\text {liq }}=$ $1396^{\circ} \mathrm{C}\left(2545^{\circ} \mathrm{F}\right)$, respectively. The heat transfer coefficient at the mold-metal interface was determined as the following function of temperature: $1100 \mathrm{~W} / \mathrm{m}^{2} \mathrm{~K}$ for temperatures above $1350{ }^{\circ} \mathrm{C}\left(2462^{\circ} \mathrm{F}\right), 200 \mathrm{~W} / \mathrm{m}^{2} \mathrm{~K}$ below $1350^{\circ} \mathrm{C}\left(2462^{\circ} \mathrm{F}\right)$, and a constant $150 \mathrm{~W} / \mathrm{m}^{2} \mathrm{~K}$ below $800^{\circ} \mathrm{C}\left(1472^{\circ} \mathrm{F}\right)$. The final thermo-physical property data sets for the Mn-steel and the olivine sand mold obtained through the present trial-and-error procedure are provided in the Ph.D. thesis of one of the authors. ${ }^{[25]}$ Examples of comparisons between measured and predicted temperatures and cooling rates at several locations are shown in Figure 4. Excellent agreement can be observed for both the temperatures in the mold and in the metal. The predictions from the thermal simulation were then used as input to the porosity model.

\section{RESULTS AND DISCUSSION}

The present porosity model is first applied to two simple test cases to illustrate its capabilities and sensitivity to changes in the adjustable model parameters. In these test cases, the evolution of the temperature and solid volume fraction fields is prescribed, i.e., no thermal simulations are performed. They show examples of how internal porosity is predicted to nucleate and grow, together with the resulting shrinkage driven flow and pressure distributions. Additional test cases that involve riser pipes and surface sinks can be found in Reference 25 . Following the presentation of these test cases, the model is applied to the experimental Mn-steel casting introduced in Section III, and the predicted porosity results are compared with observations of the porosity in the experimental castings. 


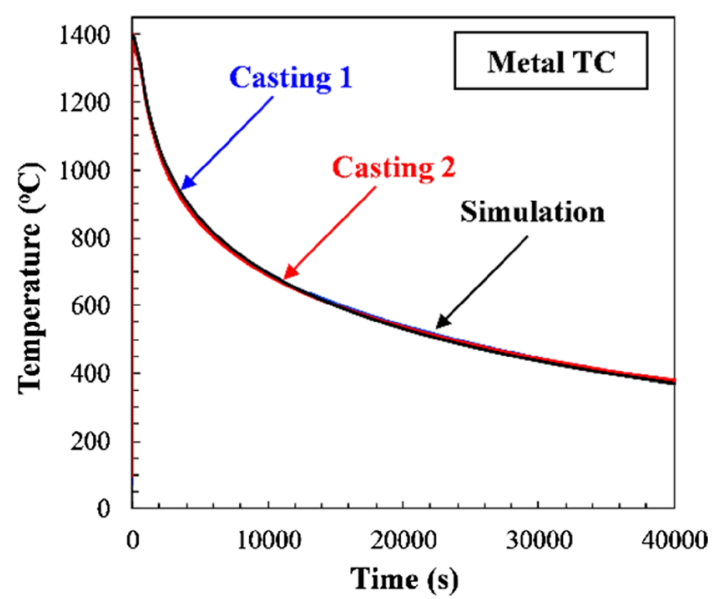

(a)

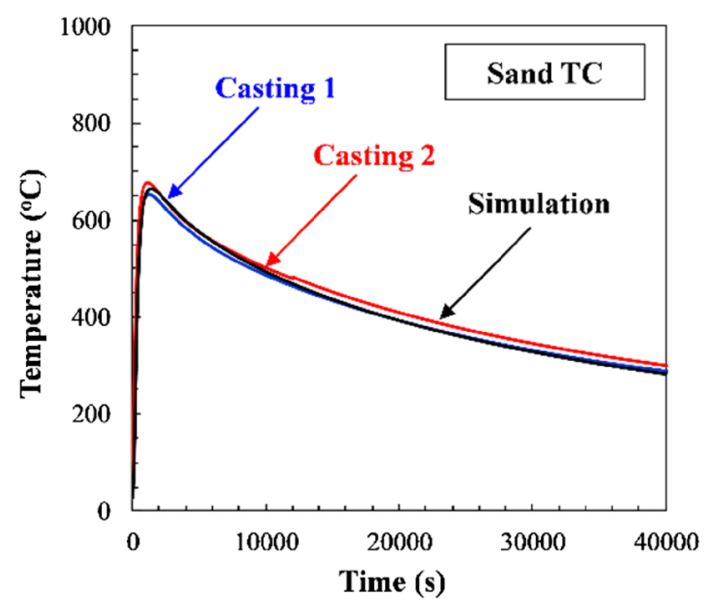

(b)

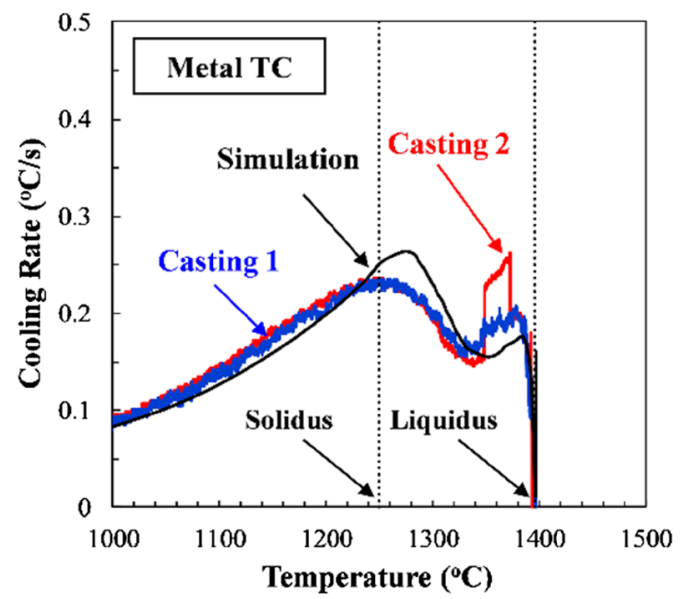

(c)

Fig. 4-Comparisons of thermocouple measurements and thermal simulation results in the metal and in the olivine sand mold: plots of (a) temperature $v s$ time at the TC_B2 location, (b) temperature vs time at the $\mathrm{TC}_{-} \mathrm{K} 3$ location, and $(\bar{c})$ cooling rate $v s$ temperature at the TC_B3 location (Color figure online).

\section{A. Model Testing}

The geometries and computational meshes for the two test case castings are shown in Figure 5. They consist of a square block and a rectangular block. The square block solidifies uniformly from the outside such that a single hot spot forms in the center, whereas the rectangular block cools such that two hot spots form. The solid fraction fields that were prescribed to produce these solidification patterns are shown in Figures 6 and 8 below. The material properties and model parameters used for the two test cases are listed in Table II. All other details can be found in the Ph.D. thesis of one of the authors. ${ }^{[25]}$

Simulation results for the first test case are shown in Figure 6. The simulation is started with the entire block in a mushy state. Figure 6(a) shows the prescribed circular solid fraction distribution at $t=1 \mathrm{~s}$, with values close to unity near the outer walls and slightly above zero in the center. At this time, a porosity containing region is predicted to be present near the center of the block where the solid fraction is the lowest (Figure 6(c)). This location is expected in the present model due to the strong effect of the solid fraction on the pore nucleation parameter $\Pi$. The pore fraction distribution is slightly skewed toward the top because of the (relatively small) influence of the hydrostatic pressure on the $\Pi$ parameter. The predicted liquid velocity vectors shown in Figure 6(b) point outward from the porosity containing region and decrease in magnitude towards the outer walls of the block. This flow feeds the solidification shrinkage of the metal in the mushy zone. The total pressure distribution in the liquid is almost hydrostatic (Figure 6(b)), because the pressure drop due to the flow is negligibly small at this stage. The smallness of the dynamic pressure variations can be attributed to the permeability still being relatively high everywhere in the block. At $t=45 \mathrm{~s}$, the solid fraction has increased to values above 0.8 everywhere in the block (Figure 6(d)). The porosity containing region has grown considerably in size (Figure 6(f)). The pore volume fraction is equal to unity in the center portion, indicating the presence of an open shrinkage hole like the one in Figure 1(b). The open hole is predicted to be surrounded by layers of distributed microporosity (see Figure 1(c)) with pore fraction values below 0.3 . These layers are now more strongly skewed towards the top because the hydrostatic pressure continues to affect the spreading of the porosity through the $\Pi$ parameter. The liquid flow around the porosity containing region has decreased considerably in magnitude. As can be seen in Figure 6(e), the total pressure distribution is no longer purely hydrostatic. With the solid fraction being close to unity, the permeability is now much lower throughout the block, causing considerable dynamic pressure variations in the mush that does not contain porosity. At $t=90 \mathrm{~s}$, the block is almost completely solidified (Figure 6(g)) and 


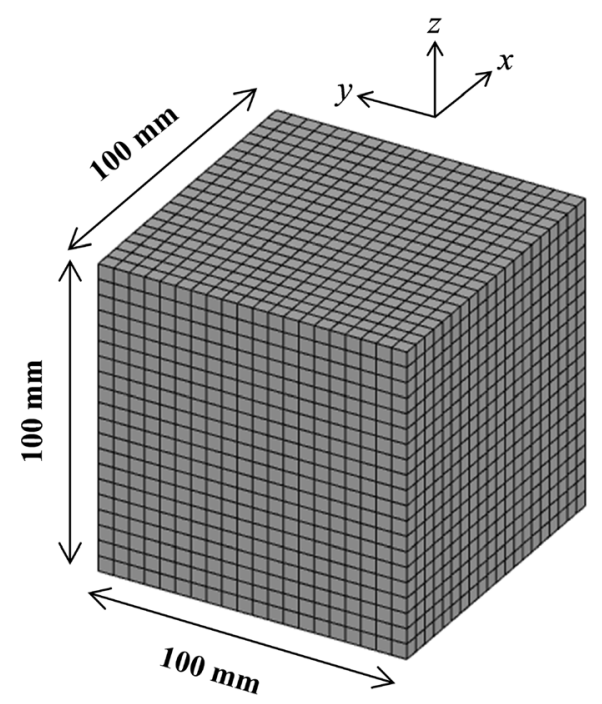

(a)

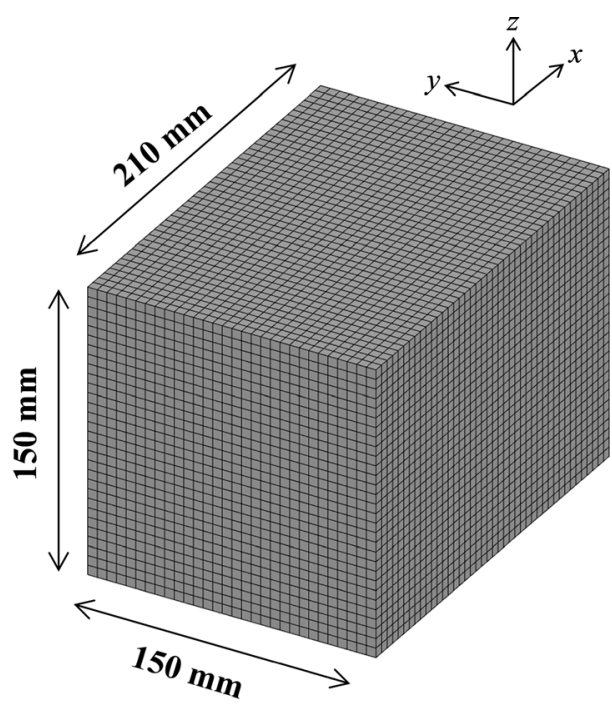

(b)

Fig. 5- Geometries of the two test cases: (a) square block with a single hot spot, and $(b)$ rectangular block with two hot spots.

the feeding velocities have vanished (Figure 6(h)). Since the liquid pressure in a 100 pct solid region is not defined, it is simply set to zero. In the part of the porosity containing region that still contains some liquid, the total pressure distribution is hydrostatic because the dynamic pressure is forced to the pore pressure, which is uniformly equal to zero for internal porosity (see Section II-A). Figure 6(i) shows the final porosity distribution predicted for the present test case casting. The microporosity that surrounds the open shrinkage hole in the center extends on average to about half the distance to the walls.

The results of a parametric study for the first test case are presented in Figure 7. In this study, the effect of changes in the model parameters $\varepsilon_{\text {layer }}, \varepsilon_{\text {nuc }}$, and $g_{\mathrm{s}, \mathrm{sur}}=$ $g_{\mathrm{s}, \mathrm{coh}}$ on the predicted final porosity pattern is investigated. Results for variations in the remaining model parameters are presented elsewhere. ${ }^{[19,25]}$ Other than for the changes noted, the parameters are held at the values given in Table II. Comparing Figures 7(a) and (b) show how $\varepsilon_{\text {layer }}$ controls the spreading of the porosity after its initial nucleation. For $\varepsilon_{\text {layer }}=0.001$, the porosity spreads non-uniformly around the center as controlled by the $\Pi$ parameter. For $\varepsilon_{\text {layer }}=0.10$, the porosity containing region appears as a more unrealistic square pattern. This can be attributed to the fact that for such a large value of $\varepsilon_{\text {layer }}$ the model activates, layer by layer, all available neighboring computational cells once the interior cells become inactive, regardless of the value of $\Pi$. The open hole in the center is similar for the two values of $\varepsilon_{\text {layer }}$ because its extent is controlled by $\varepsilon_{\text {nuc }}$. The effect of $\varepsilon_{\text {nuc }}$ can be observed in Figures 7(c) and (d). For $\varepsilon_{\text {nuc }}=0.001$, the volume in the center where porosity is initially nucleated is relatively small. This leads to the early shrinkage being distributed to fewer computational cells and, hence, to higher pore fraction values in those cells. For $\varepsilon_{\text {nuc }}=0.10$, on the other hand, more computational cells are initially nucleated and the final pore fraction values in those cells are therefore smaller. Of course, the total amount of porosity in the entire block is the same for the two cases. Variations in the critical solid fractions $g_{\mathrm{s}, \mathrm{sur}}=g_{\mathrm{s}, \mathrm{coh}}$ are investigated in Figures 7(e) and (f). For a relatively low value of $g_{\mathrm{s}, \mathrm{sur}}=g_{\mathrm{s}, \mathrm{coh}}=0.25$, the solid becomes immobile early during solidification and mass feeding is largely inhibited. This limits the maximum pore fraction in the center to values no larger than 0.75 . For $g_{\mathrm{s}, \mathrm{sur}}=g_{\mathrm{s}, \mathrm{coh}}=0.75$, however, significant mass feeding takes place and the pore fractions in the center region reach unity before the computational cells at that location reach a solid fraction of 0.75 . Even though $g_{\mathrm{s}, \mathrm{sur}}$ and $g_{\mathrm{s}, \mathrm{coh}}$ were varied simultaneously in the present parametric study, the results are only affected by $g_{\mathrm{s}, \mathrm{coh}}$. Surface porosity does not form in the present case because the simulation was only started when the solid fraction at the walls of the square block exceeded 0.75 (see Figure 6(a)).

The second test case, presented in Figure 8, is intended to demonstrate the model capabilities for multiple porosity forming regions. A time varying solid fraction field is prescribed that corresponds to two hot spots forming simultaneously. Representative solid fraction fields are presented in Figures 8(a), (d), and (g). The solid fraction field is symmetric about the vertical mid-plane of the rectangular block (see Figure 5(b)), with each half containing one hot spot at its center where the solid fraction is lowest. The term hot spot is used because a lower solid fraction generally corresponds to a higher temperature. The solid fraction gradients were prescribed to be steeper than in the first test case, as can be seen by comparing Figures 6(a) and 8(a). As shown in Figures 8(c), (f), and (i), porosity nucleates and grows in each hot spot in a manner similar to the first test case. Due to the steeper solid fraction gradients, however, the porosity around the open center 
Solid-fraction, $g_{s}(-)$


(a)


(d)


(g)
Total Pressure, $\boldsymbol{P}_{\ell, t}$ (bar)



0.0

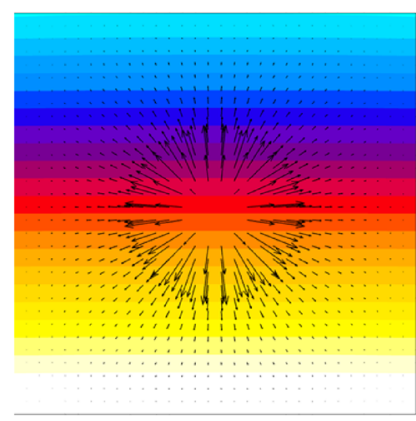

$10^{-3} \mathrm{~m} / \mathrm{s}$

(b)


$10^{-4} \mathrm{~m} / \mathrm{s}$

(e)

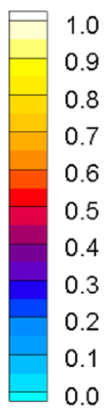

0.0

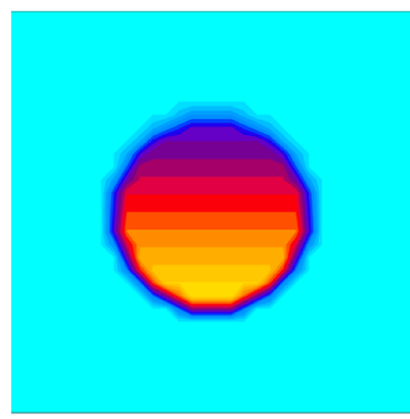

$10^{-5} \mathrm{~m} / \mathrm{s}$

(h)
Porosity, $g_{p}(-)$



0.005
0.000

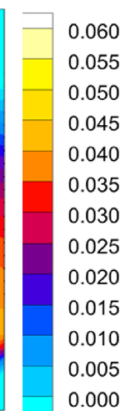

0.000

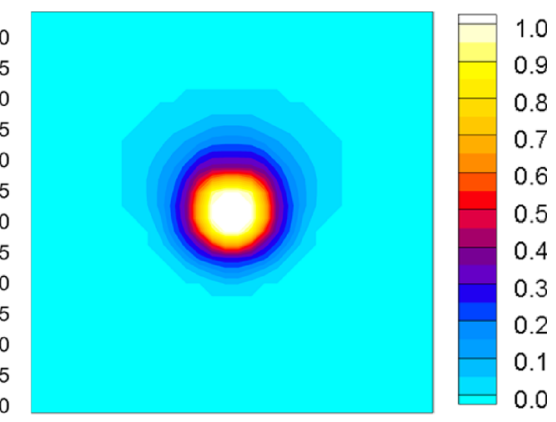

(f)

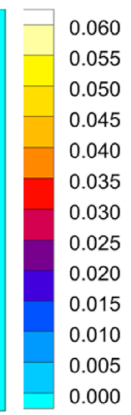

0.000

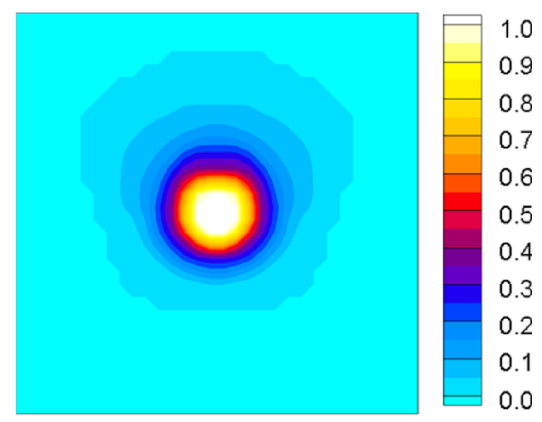

(i)

Fig. 6-Mid-plane simulation results for the first test case casting: prescribed solid fraction (first column), total pressure and liquid velocity vector (second column), and porosity (third column) distributions at $1 \mathrm{~s}[(a)$ through $(c)], 45 \mathrm{~s}[(d)$ through $(f)]$, and $90 \mathrm{~s}[(g)$ through $(i)]($ Color figure online).

hole is more concentrated and does not spread out as much as in the first test case. More importantly, Figures $8(\mathrm{~b}),(\mathrm{e})$, and (h) show that the shrinkage driven flow is calculated correctly. No feeding zones are defined in the present model, and the equations are solved in a single domain approach. Nonetheless, the flow field is properly divided at the vertical mid-plane and each of the growing porosity regions feeds the solidification shrinkage in exactly one half the domain. Due to the steep solid fraction gradient, most of the pressure drop occurs over a very thin layer along the boundary between the mushy zone and the fully solidified region.

The above two simple test cases demonstrate that, in theory, the model can predict porosity in castings with one or more hot spots in a manner that appears to be realistic. Again, additional test cases and parametric studies can be found in the Ph.D. thesis of one of the
Table II. Material Properties and Model Parameters Used in the Simulations

\begin{tabular}{ll}
\hline Parameter & \multicolumn{1}{c}{ Value } \\
\hline$K_{0}\left(\mathrm{~m}^{2}\right)$ & $1.7 \times 10^{-9}$ \\
$K_{\min }\left(\mathrm{m}^{2}\right)$ & $1 \times 10^{-16}$ \\
$K_{\max }\left(\mathrm{m}^{2}\right)$ & $1 \times 10^{-6}$ \\
$g_{\mathrm{s}, \mathrm{sur}}(-)$ & 0.55 \\
$g_{\mathrm{s}, \mathrm{coh}}(-)$ & 0.55 \\
$\varepsilon_{\text {layer }}(-)$ & $3.5 \times 10^{-2}$ \\
$\varepsilon_{\text {nuc }}(-)$ & $5 \times 10^{-3}$ \\
$P_{\text {atm }}(\mathrm{bar})$ & 1.01325 \\
$P_{\sigma, 0}(\mathrm{bar})$ & $P_{\text {atm }}$ \\
$P_{\text {mold }}(\mathrm{bar})$ & $0.9 P_{\text {atm }}$ \\
$\rho_{\mathrm{s}}\left(\mathrm{kg} / \mathrm{m}^{3}\right)$ & 7124 \\
$\rho_{\ell}\left(\mathrm{kg} / \mathrm{m}^{3}\right)$ & 6894 \\
$\mu_{\ell}(\mathrm{Pa} \mathrm{s})$ & $5.63 \times 10^{-3}$ \\
\hline
\end{tabular}




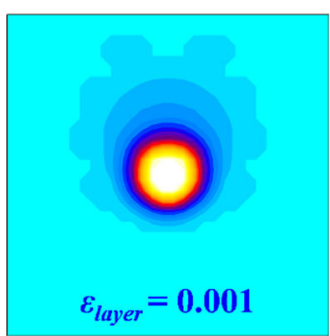

(a)

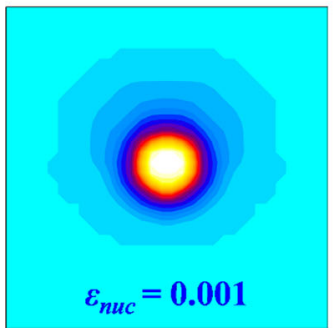

(c)



(e)

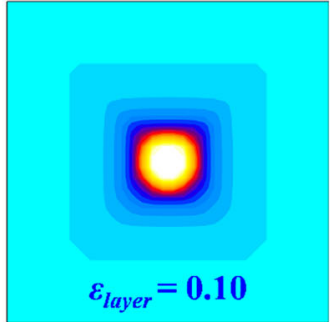

(b)

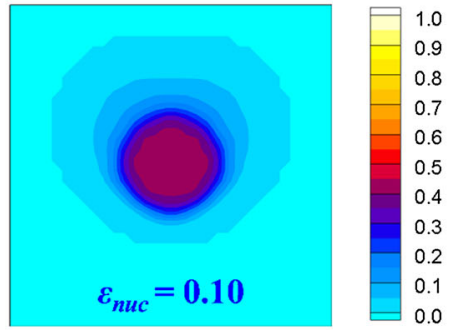

(d)

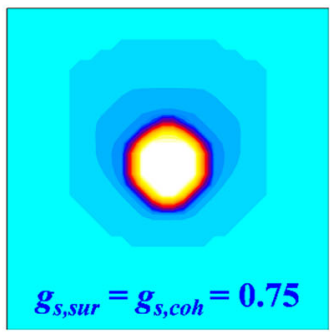

(f)
Fig. 7-Results of a parametric study for the first test case casting: predicted final porosity patterns at mid-plane for $(a) \varepsilon_{\text {layer }}=0.001$, (b) $\varepsilon_{\text {layer }}=0.1$, (c) $\varepsilon_{\text {nuc }}=0.001$, (d) $\varepsilon_{\text {nuc }}=0.1$, (e) $g_{\mathrm{s}, \text { sur }}=g_{\mathrm{s}, \mathrm{coh}}=$ 0.25 , and $(f) g_{\mathrm{s} \text {, sur }}=g_{\mathrm{s} \text {, coh }}=0.75$; all other parameters are held at the values in Table II (Color figure online).

authors. ${ }^{[25]}$ It is shown that the predictions can be tuned by changes in the model parameters to conform closely to reality. Such tuning was demonstrated for an aluminum alloy casting in Reference 19, but in that study the feeding flow was not calculated. Model tuning for the experimental $\mathrm{Mn}$-steel casting of the present study is demonstrated in the next sub-section.

\section{B. Model Application to the Mn-Steel Casting}

Next, the present porosity model is used to simulate the Mn-steel casting of Section III. The model parameters used in the simulation are those listed in Table II, while the results of the simulation are shown in Figures 9 and 10. Figure 9 shows results on a vertical plane that cuts through the center of the gating system and the riser, while Figure 10 is for a mid-width plane through the casting. The first column in each figure depicts the evolution of the solid fraction field from the thermal simulation, the second column the total pressure and velocity vector fields, and the third column the pore fraction distribution. The various fields are provided at four different times up to 1650 seconds, which is close to the final solidification time of the casting. Until almost 150 seconds, all shrinkage of the metal during cooling and solidification is fed by the gating system. Metal flows from the pouring cup through the sprue and the runner into the casting (Figures 9(b) and (e)). As a result of that flow, a large cavity forms in the pouring cup and the upper portion of the sprue (Figure 9(f)). The total pressure distribution is mostly hydrostatic, because the dynamic pressure variations are still very small. The pressure is hydrostatic even in the end block that is separated from the riser through a relatively long and thin plate section (Figure 10(e)), because there is still a path through the plate section where the solid fraction is less than 0.7 (Figure 10(d)). Shortly before 150 seconds, a small shrinkage hole is predicted to form at the top of the riser (Figures 9(f) and 10(f)). The top of the riser is connected to the atmosphere through a small vent that induces the shrinkage pipe at that location once the pressure falls below atmospheric pressure. The gating system solidifies completely at about 300 seconds, (Figure 9(g)), after which the shrinkage is fed mostly by the downward growing hole at the top of the riser (Figure 9(i)). The feeding flow spreads from the growing riser pipe into all solidifying portions of the casting (Figure 9(e) and (h); Figure 10(e) and (h)). At 300s, the pressure is starting to experience a significant drop in the plate section (Figure 10(h)), because the solid fraction is now above 0.9 throughout its thickness (Figure 10(g)). Eventually, the pressure in the end block reaches vacuum and porosity nucleates in its center where the solid fraction is lowest (Figure 10(i)). This porosity now feeds the shrinkage in the end block and the right portion of the plate section. The casting is almost completely solidified at 1650 seconds (Figures 9(j) and $10(\mathrm{j})$ ). The shrinkage holes in the riser and in the end block have grown to considerable size (Figures 9(1) and 10(1)). These open holes are surrounded by smaller microporosity fields. Additional microporosity, with pore fraction values below 0.1 , is predicted to form late in the solidification process throughout most of the plate section (Figure 10(1)).

A comparison between the predicted and experimentally observed porosity is shown in Figure 11. First, the gating system was removed, and the casting was X-rayed from the top. Then, the casting was cut in half at mid-width. Finally, a thin slice (about $7 \mathrm{~mm}$ thick) was cut from one of the halves adjacent to the first cut. The cut surfaces from the intact half and from the side of the slice opposite to the first cut were subjected to dye penetrant testing to reveal microporosity. Images of these two surfaces, located at or near mid-width, are provided in Figure 11(a), together with the simulation result at mid-width. Even though the open riser pipe in the experiment is very irregular in shape, its overall width and depth are predicted well. The microporosity emanating from the bottom of the riser pipe is also seen in the simulation result, but its extent into the steps adjacent to the riser is somewhat underpredicted. On the other hand, the microporosity revealed by the dye penetrant testing in the thin plate section appears to be well reproduced by the simulation. A close-up of the mid-width results for the end block is displayed in Figure 11(b). The open shrinkage hole in the center and 
Solid-fraction, $g_{s}(-)$

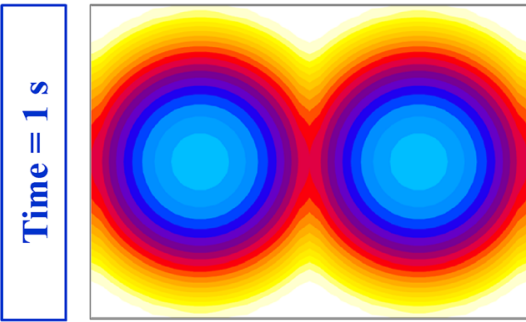

(a)

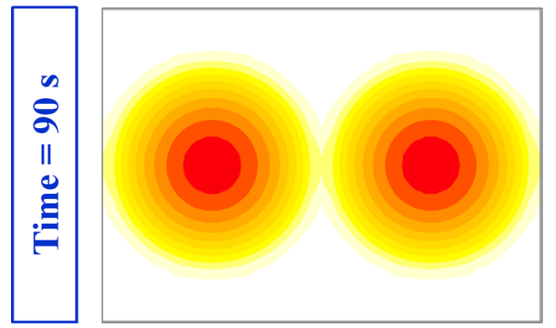

(d)

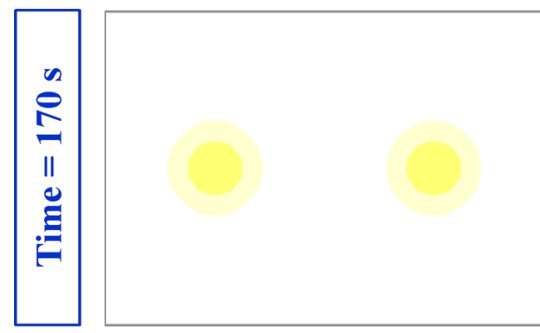

(g)
Total Pressure, $\boldsymbol{P}_{\ell, t}$ (bar)



(1)

$10^{-3} \mathrm{~m} / \mathrm{s}$


$5 \times 10^{-4} \mathrm{~m} / \mathrm{s}$

(e)
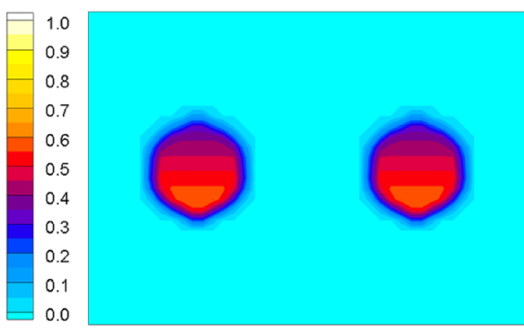

$10^{-5} \mathrm{~m} / \mathrm{s}$

(h)
Porosity, $g_{p}(-)$
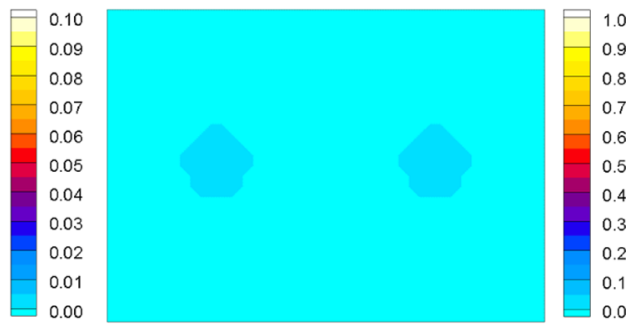

(c)
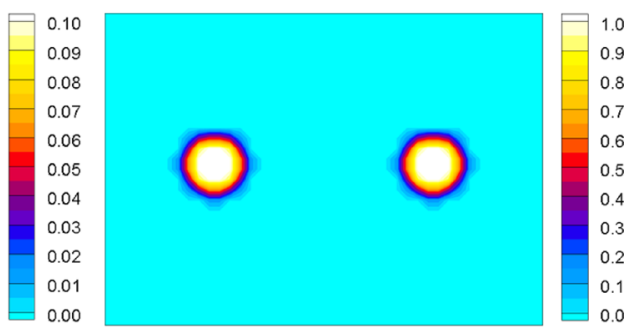

(f)
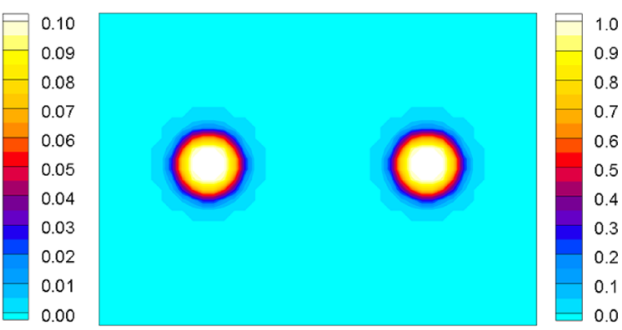

(i)

Fig. 8-Mid-plane simulation results for the second test case casting: prescribed solid fraction (first column), total pressure and liquid velocity vector (second column), and porosity (third column) distributions at $1 \mathrm{~s}[(a)$ through $(c)], 90 \mathrm{~s}[(d)$ through $(f)]$, and $170 \mathrm{~s}[(g)$ through $(i)]($ Color figure online).

the surrounding microporosity are both predicted very well. It should be noted that the more gradual variation in the predicted pore fraction values at the edge of the open hole is simply due to the interpolation algorithm used in plotting the results. An X-ray image of the end block, taken from the top, is shown in Figure 11(c). The corresponding simulation result was obtained by averaging the predicted pore fractions through the thickness of the block. Again, good overall agreement can be observed for the shrinkage hole at the center.

The above comparison was performed after an extensive computational study where the adjustable parameters in the porosity model were varied. ${ }^{[25]}$ The values of the model parameters listed in Table II are the result of this study and represent optimum values that give the best overall agreement with the experimental observations. It is, nonetheless, instructive to examine some of the results from the parametric study, as shown in Figure 12, in order to gain more insight into the sensitivity of the model to variations in the parameters. The shrinkage pipe in the riser for $\varepsilon_{\text {layer }}=0.01$ is too large at the bottom, whereas for $\varepsilon_{\text {layer }}=0.1$ it is too wide at the top (Figure 12(a)). In addition, for $\varepsilon_{\text {layer }}=0.1$ the pore fraction values in the center of the end block are too low. Changing $\varepsilon_{\text {nuc }}$ from 0.01 to 0.1 increases the size of the open shrinkage hole in the end block, while the predicted riser pipe is relatively unaffected (Figure 12(b)). Increasing $g_{\mathrm{s}, \mathrm{sur}}=g_{\mathrm{s}, \mathrm{coh}}$ from 0.45 to 0.6 also has the largest effect on the porosity in the end block (Figure 12(c)). As explained in connection with Figure 7, a larger coherency solid fraction causes the shrinkage hole in the center to be more open. The effect of the permeability coefficient $K_{0}$ is investigated in Figure $12(\mathrm{~d})$. A small $K_{0}$ of $5 \times 10^{-10}$ results in microporosity, with pore fraction values below 0.1 , being predicted throughout most of the casting. This can be 
Solid-fraction, $g_{s}(-)$
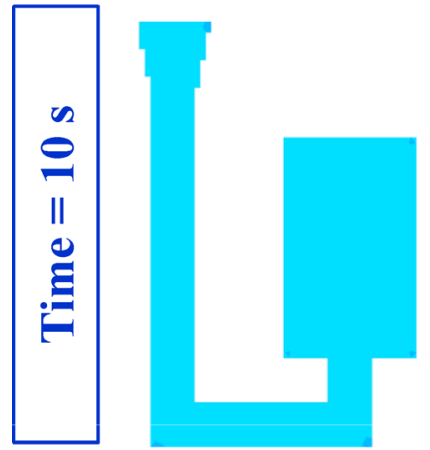

(a)
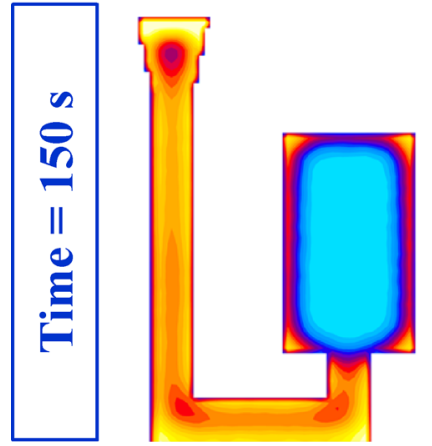

(d)
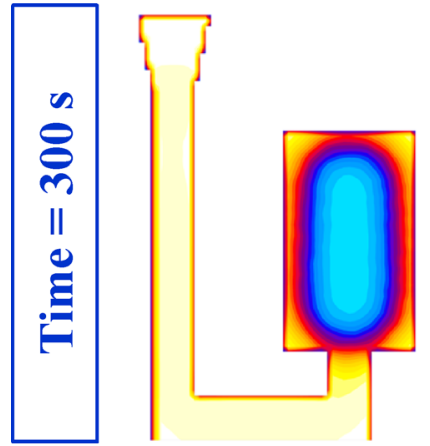

(g)
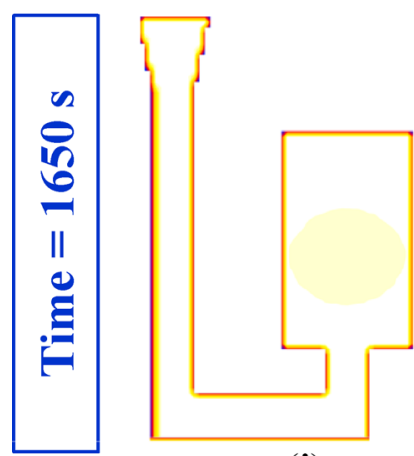

(j)
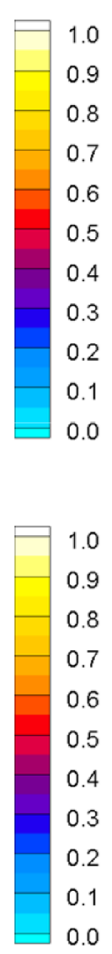

0.0

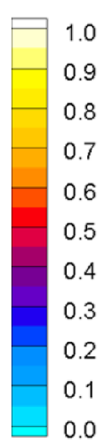

0.0

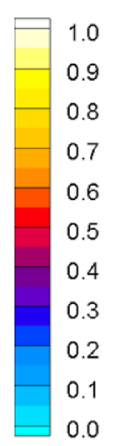

0.0

\section{Total Pressure, $\boldsymbol{P}_{\ell, t}$ (bar)}
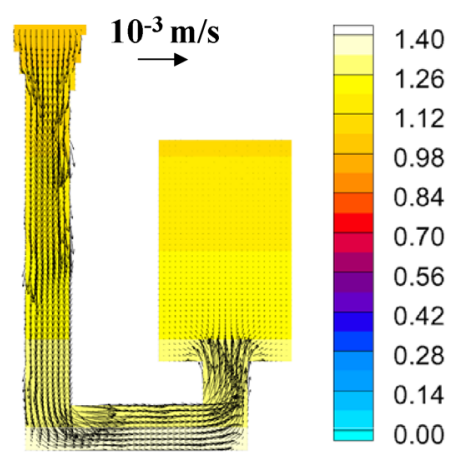

(b)

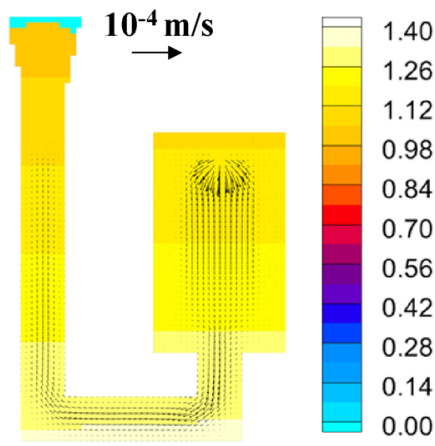

(e)

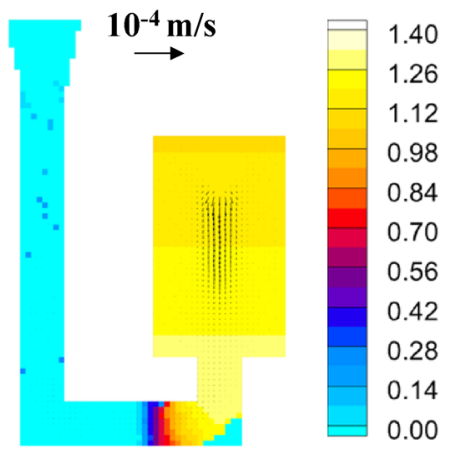

(h)

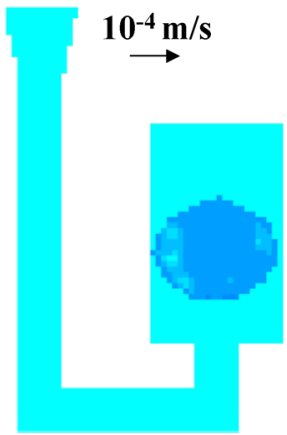

(k)

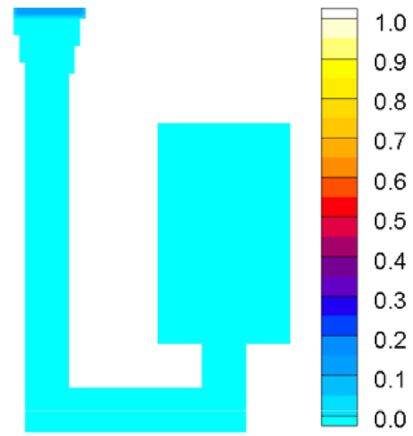

(c)

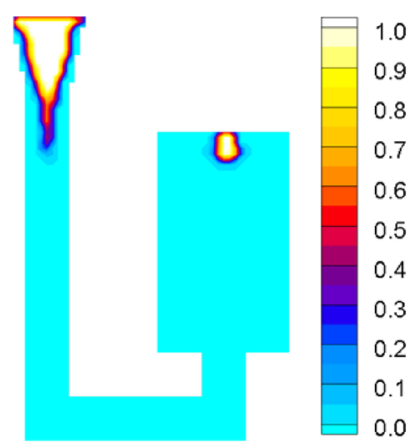

(f)

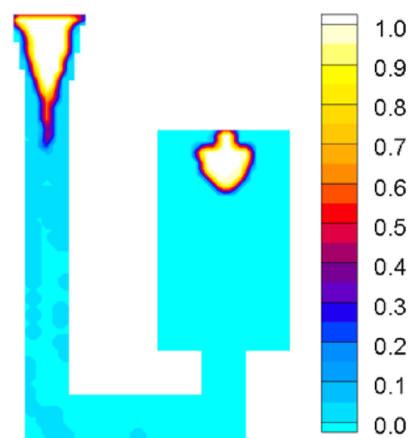

(i)

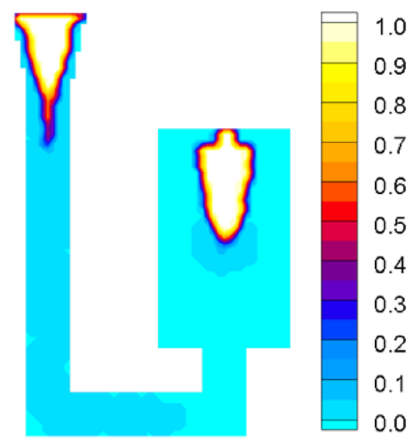

(I)

Fig. 9-Simulation results for the Mn-steel casting at a vertical plane cutting through the middle of the riser and the gating system: solid fraction (first column), total pressure and liquid velocity vector (second column), and porosity (third column) distributions at $10 \mathrm{~s}[(a)$ through $(c)], 150 \mathrm{~s}[(d)$ through $(f)], 300 \mathrm{~s}[(g)$ through $(i)]$, and $1650 \mathrm{~s}[(j)$ through $(l)]$ (Color figure online). 


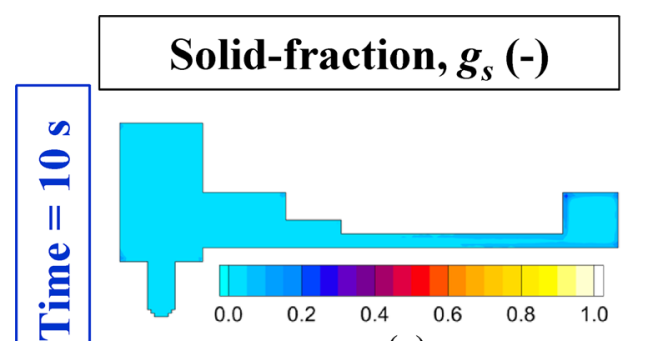

(a)
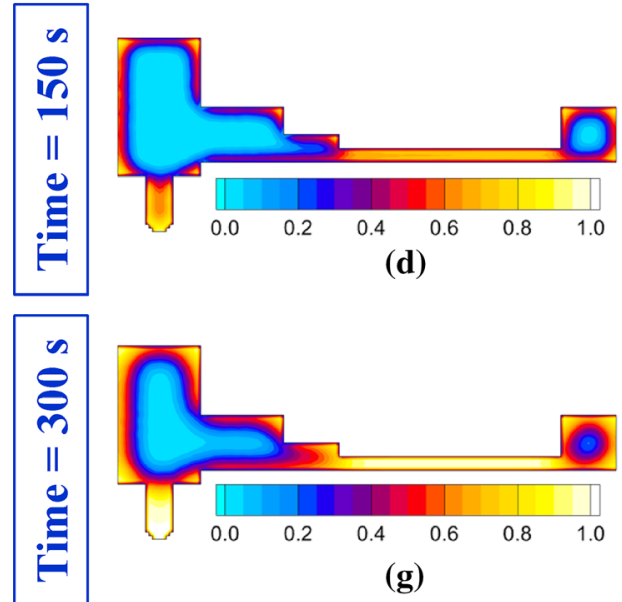

(g)

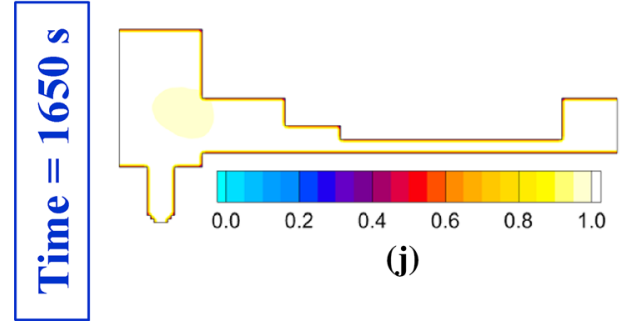

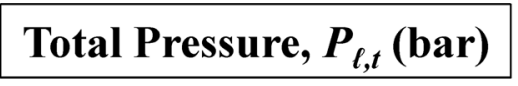

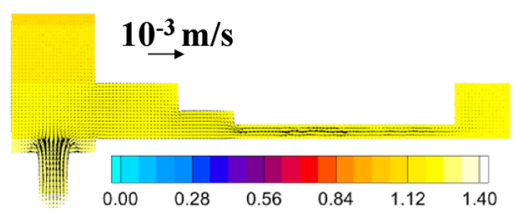

(b)

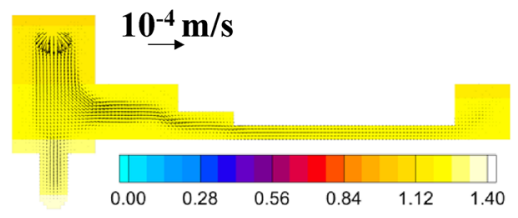

(e)

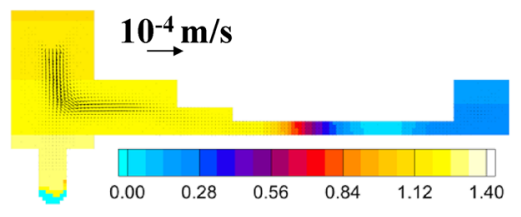

(h)

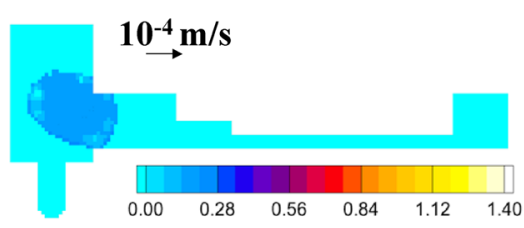

(k)

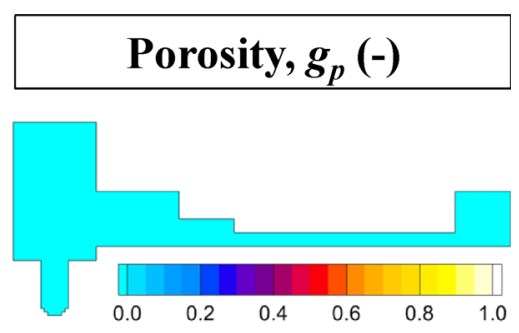

(c)
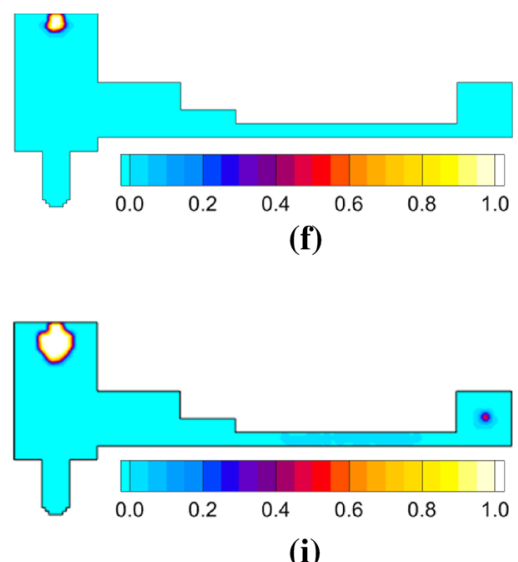

(i)

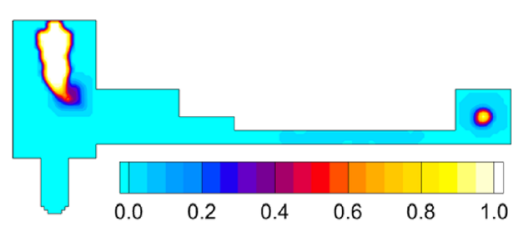

(I)

Fig. 10-Simulation results for the Mn-steel casting at a vertical plane cutting through the casting at mid-width: solid fraction (first column), total pressure and liquid velocity vector (second column), and porosity (third column) distributions at $10 \mathrm{~s}[(a)$ through $(c)]$, $150 \mathrm{~s}[(d)$ through $(f)], 300 \mathrm{~s}[(g)$ through $(i)]$, and $1650 \mathrm{~s}[(j)$ through $(l)]$ (Color figure online).

attributed to the pressure dropping to lower values more rapidly, inducing porosity to nucleate late in solidification almost everywhere. Due to more of the shrinkage being present in the form of microporosity, the shrinkage hole in the end block becomes too small. For an unrealistically large permeability coefficient of $5 \times 10^{-8}$, on the other hand, the microporosity in the thin plate section disappears and the shrinkage hole in the end block becomes too large. Changing $K_{\min }$ from $1.5 \times$ $10^{-21}$ to $1.5 \times 10^{-14}$ has a profound on the predicted porosity distribution (Figure 12(e)). For the lower value, the porosity field is almost identical to the one for the optimum value of $K_{\min }=1 \times 10^{-16}$. For the higher value, however, the shrinkage pipe in the riser is much too large, the microporosity in the plate section vanishes, and the pore fractions in the end block are much too low. This can be attributed to the pressure never reaching low enough values for porosity to evolve in the plate section and the end block. The changes in $P_{\text {mold }}$ explored in Figure 12(f) have a relatively small effect on the predicted porosity, in that only minor variations in the shape of the shrinkage pipe in the riser can be observed. Increasing $P_{\sigma, 0}$ from $0.5 P_{\text {atm }}$ to $2 P_{\text {atm }}$ also affects primarily the shape of the riser pipe (Figure 12(g)). For $P_{\sigma, 0}=0.5 P_{\mathrm{atm}}$, the pipe is too large at the top of the riser, whereas for $P_{\sigma, 0}=2 P_{\text {atm }}$ it is too 


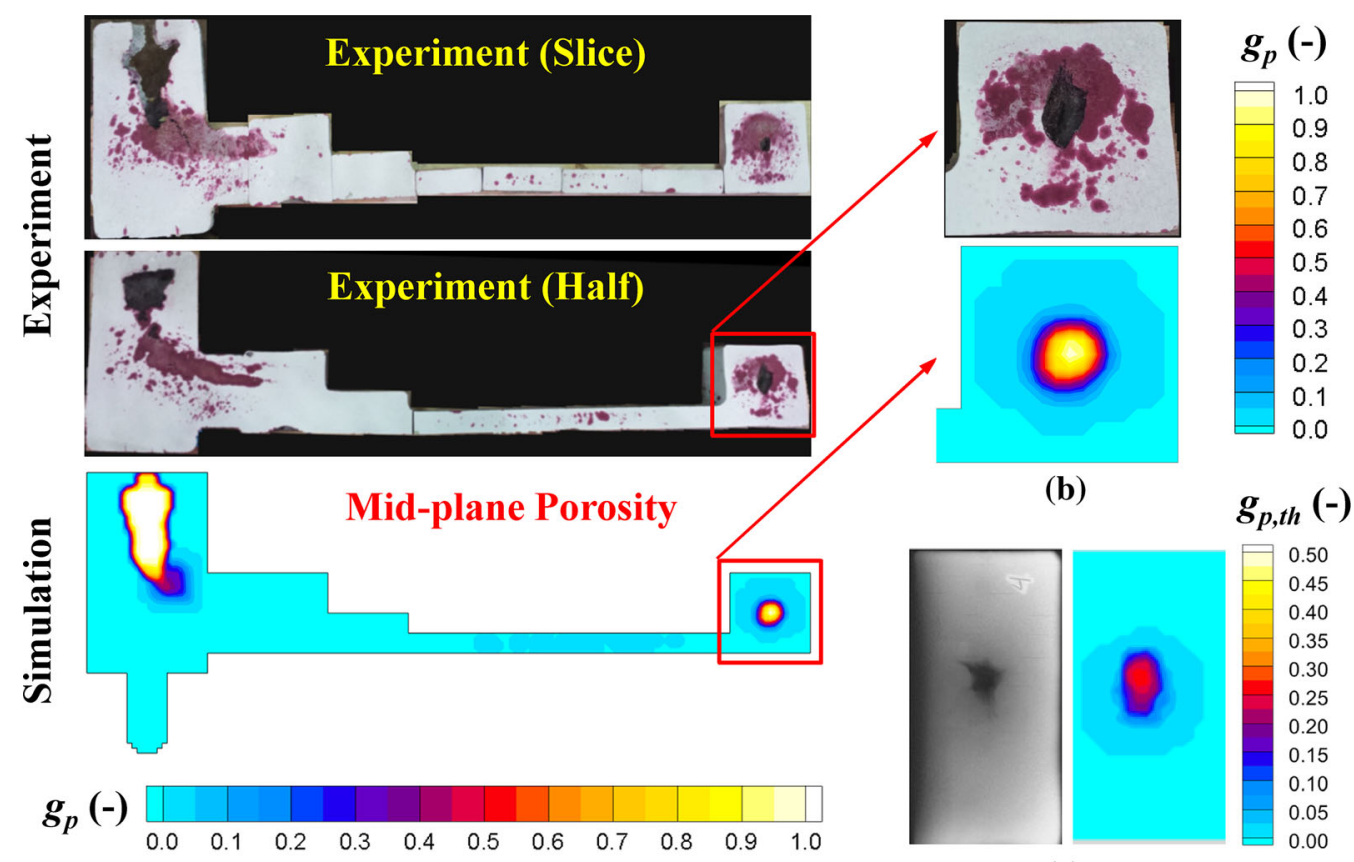

(a)

(c)

Fig. 11 - Comparison of measured and predicted porosity distributions in the Mn-steel casting: (a) vertical slices through the casting at or near mid-width, with the red areas in the experimental images obtained from dye penetrant testing; $(b)$ close-up of the porosity in the end block at mid-width; (c) top-view radiograph of the end block and through-thickness averaged predicted porosity (Color figure online).

wide at about mid-height where the pipe ends. This can be attributed to the fact that the effect of the hydrostatic pressure in the $\Pi$ parameter becomes larger for decreasing $P_{\sigma, 0}$.

\section{CONCLUSIONS}

A model for the prediction of shrinkage porosity in metal castings is developed. It is based on solutions of the basic equations for the feeding flow and pressure distribution during solidification and an extended version of the pore nucleation and growth model of Khalajzadeh et al. ${ }^{[19]}$ The model uses the temperature and solid fraction results from a thermal simulation and predicts all kinds of shrinkage porosity, including surface sinks and riser pipes, open shrinkage holes internal to the casting, and microporosity dispersed between dendrite arms. The governing equations are solved in a single domain approach in which multiple porosity regions are considered simultaneously without defining separate feeding zones. The model contains several adjustable parameters, namely $\varepsilon_{\text {nuc }}, \varepsilon_{\text {layer }}, g_{\mathrm{s}, \mathrm{sur}}$, $g_{\mathrm{s}, \mathrm{coh}}, K_{0}, K_{\min }, P_{\text {mold }}$, and $P_{\sigma, 0}$. The effect of these parameters on the predicted porosity distribution is investigated in parametric studies. For an optimum combination of these parameters, good agreement is obtained with observations made in an experimental Mn-steel sand casting. The shape of the riser pipe, the microporosity in a thin plate section, and the open shrinkage hole with surrounding microporosity in the thermal center of an end block are all predicted correctly. More quantitative comparisons between measured and predicted pore fraction values can be found in Khalajzadeh et al. ${ }^{[9,25]}$ It should be noted that the optimum values of the adjustable parameters found here are very similar to the ones obtained for the aluminum alloy casting investigated by Khalajzadeh et al., ${ }^{[19]}$ except that the two permeability parameters are new 

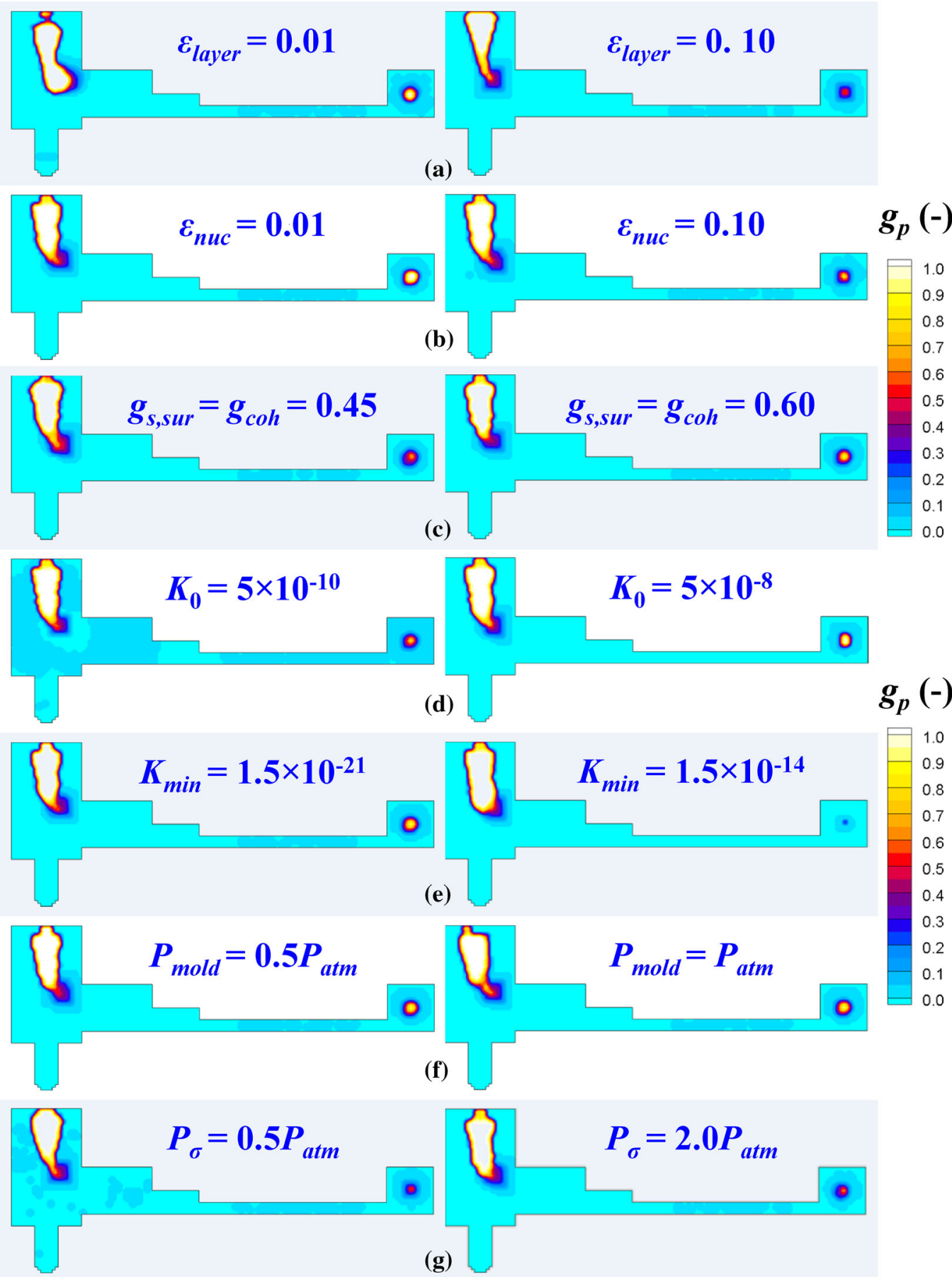

Fig. 12-Results of a parametric study for the Mn-steel casting at a vertical plane cutting through the casting at mid-width: predicted final porosity distributions for different values of $(a) \varepsilon_{\text {layer }},(b) \varepsilon_{\text {nuc }},(c) g_{\text {s, sur }}=g_{\text {s,coh }},(d) K_{0},(e) K_{\text {min }},(f) P_{\text {mold }}$, and $(g) P_{\sigma, 0}($ Color figure online $)$.

to this study. This provides a first indication that the adjustable parameters are not too sensitive to the type of alloy. Some changes can be expected, for example, in the coherency solid fraction when a fully columnar microstructure forms during solidification; the Mn-steel of the present study and the aluminum alloy of Reference 19 were fully equiaxed. Application of the model to different metal alloys and different types of 
casting processes (e.g., permanent mold, investment, etc.) is needed to further validate the predictions and establish a reliable set of adjustable parameters.

\section{REFERENCES}

1. M.J. Couper, A.E. Neeson, and J.R. Griffiths: Fatigue Fract. Eng. Mater. Struct., 1990, vol. 13, pp. 213-27.

2. Q.G. Wang, D. Apelian, and D.A. Lados: J. Light Met., 2001, vol. 1, pp. 73-84.

3. R.A. Hardin and C. Beckermann: Metall. Mater. Trans. A, 2013, vol. 44A, pp. 5316-32.

4. T.S. Piwonka and M.C. Flemings: Trans. AIME, 1966, vol. 236, pp. $1157-65$.

5. K. Kubo and R.D. Pehlke: Metall. Trans. B, 1985, vol. 16B, pp. 359-66.

6. C. Pequet, M. Gremaud, and M. Rappaz: Metall. Mater. Trans. A, 2002, vol. 33A, pp. 2095-16.

7. K.D. Carlson, Z. Lin, R.A. Hardin, and C. Beckermann: 56th Steel Founders Society of America Technical and Operating Conference, Paper No. 4.4, Steel Founders' Society of America, Chicago, IL, 2002.

8. K.D. Carlson, Z. Lin, and C. Beckermann: Metall. Mater. Trans. $B, 2007$, vol. 38 B, pp. 541-55.

9. K.D. Carlson and C. Beckermann: Metall. Mater. Trans. A, 2009, vol. 40A, pp. 163-75.

10. Y. Awano and K. Morimoto: Int. J. Cast Met. Res., 2004, vol. 17, pp. 107-14.
11. P.D. Lee and J.D. Hunt: Acta Mater., 1997, vol. 45, pp. 4155-69.

12. R.C. Atwood, S. Sridhar, W. Zhang, and P.D. Lee: Acta Mater., 2000, vol. 48 , pp. 405-17.

13. L. Arnberg and R. Mathiesen: JOM, 2007, vol. 59, pp. 20-26.

14. A. Reis, Y. Houbaert, Z. Xu, R. Van Tol, A.D. Santos, J.F. Duarte, and A.B. Magalhaes: J. Mater. Process. Technol., 2008, vol. 202, pp. 428-34.

15. A. Reis, Z. Xu, R.V. Tol, and R. Neto: J. Manuf. Process., 2012, vol. 14 , pp. 1-7.

16. P.D. Lee, A. Chirazi, and D. See: J. Light Met., 2001, vol. 1, pp. $15-30$.

17. D.M. Stefanescu: Int. J. Cast Met. Res., 2005, vol. 18, pp. 129-43.

18. P. Yousefian and M. Tiryakioğlu: Metall. Mater. Trans. A, 2018, vol. 49A, pp. 563-75.

19. V. Khalajzadeh, D.D. Goettsch, and C. Beckermann: Metall. Mater. Trans. A, 2019, vol. 50A, pp. 757-71.

20. H. Samet and M. Tamminen: IEEE Trans. Pattern Anal. Mach. Intell., 1988, vol. 10 (4), pp. 579-86.

21. MAGMAsoft: MAGMA GmbH, Aachen, Germany.

22. K.D. Carlson and C. Beckermann: Int. J. Cast Met. Res., 2012, vol. 25 , pp. $75-92$.

23. J. Miettinen, S. Louhenkilpi, H. Kytönen, and J. Laine: Math. Comput. Simul., 2010, vol. 80, pp. 1536-50.

24. JMatPro: Sente Software Ltd., Surrey Technology Centre, Surrey, United Kingdom.

25. V. Khalajzadeh: Ph.D. Thesis, University of Iowa, Iowa City, IA, 2018.

Publisher's Note Springer Nature remains neutral with regard to jurisdictional claims in published maps and institutional affiliations. 